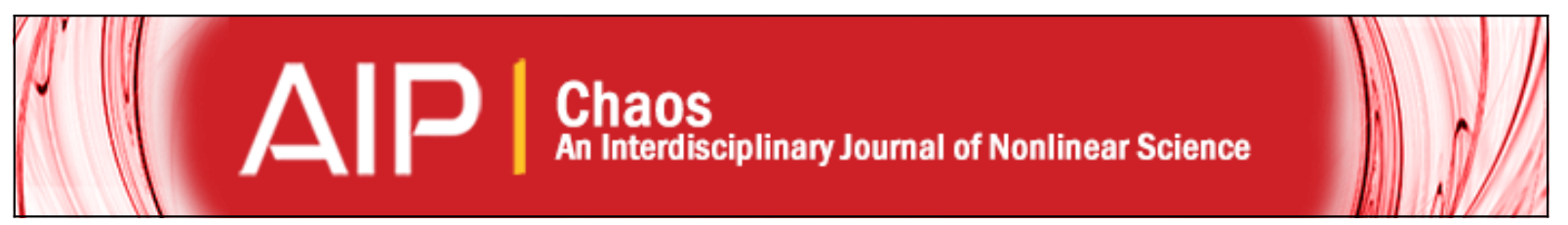

\title{
How well-connected is the surface of the global ocean?
}

Gary Froyland, Robyn M. Stuart, and Erik van Sebille

Citation: Chaos: An Interdisciplinary Journal of Nonlinear Science 24, 033126 (2014); doi: 10.1063/1.4892530

View online: http://dx.doi.org/10.1063/1.4892530

View Table of Contents: http://scitation.aip.org/content/aip/journal/chaos/24/3?ver=pdfcov

Published by the AIP Publishing

\section{Articles you may be interested in}

Simulation of the ocean's spectral radiant thermal source and boundary conditions

AIP Conf. Proc. 1531, 947 (2013); 10.1063/1.4804928

Perturbation bounds for quantum Markov processes and their fixed points

J. Math. Phys. 54, 032203 (2013); 10.1063/1.4795112

Optimal and suboptimal networks for efficient navigation measured by mean-first passage time of random walks Chaos 22, 043129 (2012); 10.1063/1.4768665

Ocean acidification and its impact on ocean noise: Phenomenology and analysis

J. Acoust. Soc. Am. 128, EL137 (2010); 10.1121/1.3431091

Identifying almost invariant sets in stochastic dynamical systems

Chaos 18, 023122 (2008); 10.1063/1.2929748

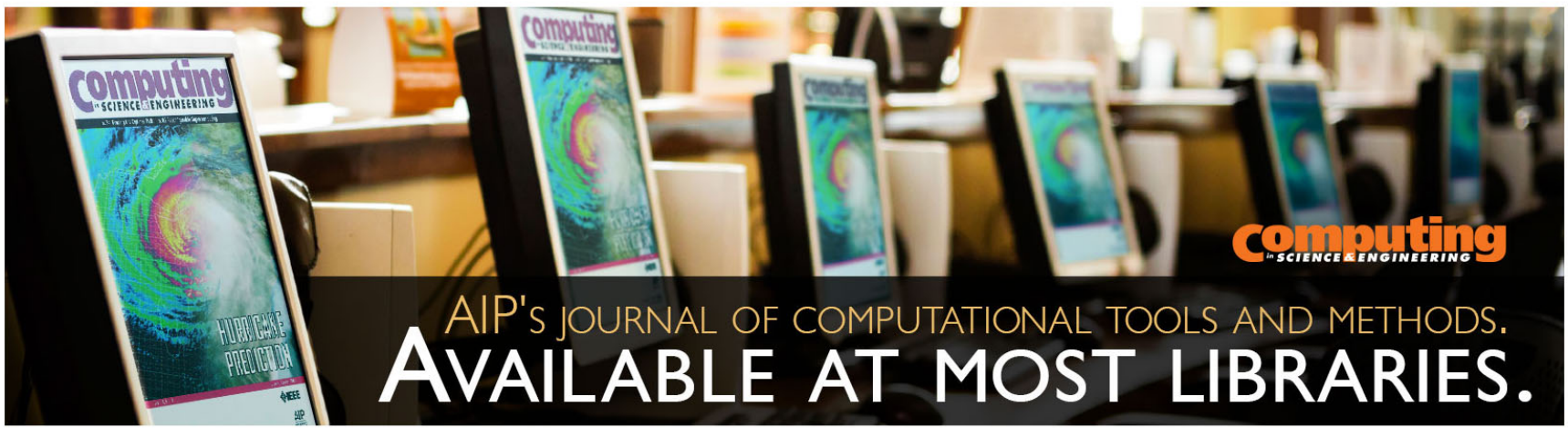




\title{
How well-connected is the surface of the global ocean?
}

\author{
Gary Froyland, ${ }^{1}$ Robyn M. Stuart, ${ }^{1}$ and Erik van Sebille ${ }^{2}$ \\ ${ }^{1}$ School of Mathematics and Statistics, The University of New South Wales, Sydney, NSW 2052, Australia \\ ${ }^{2}$ Climate Change Research Centre and ARC Centre of Excellence for Climate System Science, \\ The University of New South Wales, Sydney, NSW 2052, Australia
}

(Received 14 April 2014; accepted 28 July 2014; published online 2 September 2014)

\begin{abstract}
The Ekman dynamics of the ocean surface circulation is known to contain attracting regions such as the great oceanic gyres and the associated garbage patches. Less well-known are the extents of the basins of attractions of these regions and how strongly attracting they are. Understanding the shape and extent of the basins of attraction sheds light on the question of the strength of connectivity of different regions of the ocean, which helps in understanding the flow of buoyant material like plastic litter. Using short flow time trajectory data from a global ocean model, we create a Markov chain model of the surface ocean dynamics. The surface ocean is not a conservative dynamical system as water in the ocean follows three-dimensional pathways, with upwelling and downwelling in certain regions. Using our Markov chain model, we easily compute net surface upwelling and downwelling, and verify that it matches observed patterns of upwelling and downwelling in the real ocean. We analyze the Markov chain to determine multiple attracting regions. Finally, using an eigenvector approach, we (i) identify the five major ocean garbage patches, (ii) partition the ocean into basins of attraction for each of the garbage patches, and (iii) partition the ocean into regions that demonstrate transient dynamics modulo the attracting garbage patches. (C) 2014 AIP Publishing LLC.
\end{abstract}

[http://dx.doi.org/10.1063/1.4892530]

Ocean dynamics operate and affect climate on timescales of months to millenia. In this paper, we investigate phenomena on the ocean's surface that manifest over very long time periods: we look for regions in which water, biomass, and pollutants become trapped "forever" (which we refer to as attracting regions), or for long periods of time before eventually exiting (which we refer to as almostinvariant regions). While attracting regions may be quite small in size or irregular in shape, they can nonetheless exert great influence on the global ocean surface dynamics if their basins of attraction are large.

\section{INTRODUCTION}

In this paper, we study how well water mixes between different regions of the surface ocean. A better understanding of the surface ocean's mixing properties might help us study the evolution of the so-called great ocean garbage patches, ${ }^{1-4}$ which are regions in which plastics and other floating debris accumulate after being carried there by winds and currents.

Several recent papers have analyzed almost-invariant regions in oceans, ${ }^{5-7}$ but less attention has been devoted to analyzing attracting regions and their basins of attraction. One study in this direction is Kazantsev, ${ }^{8,9}$ who identified low-period orbits for a barotropic ocean model on a square.

In this paper, we take a probabilistic approach, which is based on analyzing a spatial discretisation of the flow dynamics. We take a set of short-run trajectories from a global ocean model and use these to construct a transition matrix, thereby representing the dynamics as a Markov chain (see Refs. 4-6); a related approach, which may be viewed as a time-derivative of this construction, is discussed in Refs. 10 and 11 . The transition matrix enables us to efficiently compute the evolution of densities and to calculate surface upwelling and downwelling. We are also able to make probabilistic statements about the flow; in particular, we can define the probability of eventual absorption into an attracting region from any other region on the surface ocean.

We identify attracting regions of the surface ocean and combine these results with an eigenvector approach to partition the ocean into regions that interact minimally with other regions. The transfer operator methods developed in Ref. 12 reveal the locations of the ocean garbage patches. We further adapt these methods to decompose the surface ocean into almost-invariant sets in a forward-time and backward-time sense. We are also able to interpret the backward-time partitioning as a decomposition of the surface ocean into basins of attraction of the major garbage patches.

Our modelling framework allows for the possibility that particles of water may exit the ocean's surface by washing up on coastlines or being absorbed into the polar sea ice. Dynamically speaking, we have an open dynamical system. Open dynamical systems theory has been developed to handle a wide variety of problems in which there is some probability of trajectories exiting the domain; we refer the reader to the recent survey. ${ }^{13}$ Our approach also allows us to determine the probability that a particle in any given location will eventually leave the computational domain.

An outline of this paper is as follows. In Sec. II, we describe the data and a method to discretize the dynamics. We use this method to calculate the forward evolution of trajectories and surface up- and downwelling; these results are contained in Sec. III. In Sec. IV, we define attracting sets, basins of attraction, and absorption probabilities for the 
discretized dynamics. In Sec. V, we describe our main procedure for analyzing connectivity, based on a transfer operator method of constructing almost-invariant sets and basins of attraction. Section VI concludes the article.

\section{DATA DESCRIPTION, DEFINITIONS, AND METHOD}

Let $\bar{X}$ denote the entire surface of the ocean, considered to be a compact two-dimensional manifold. Denote by $\bar{T}(x)$ the terminal point of a trajectory beginning at $x \in \bar{X}$ and integrated forward over 48 weeks. While this period does not resolve the entire annual cycle, it does resolve most of the variability in the ocean flow. Furthermore, the mean-flow dynamics of interest here is relatively insensitive to variability in the flow. This is apparent in the agreement between the location and evolution of the garbage patches as presented here (using 48 weeks of data), in time-mean analysis ${ }^{3}$ and in analysis including a seasonal cycle. ${ }^{4}$

We use the (time-dependent) horizontal velocity vector fields taken from the Ocean General Circulation Model for the Earth Simulator (OFES model). OFES is a global highresolution ocean-only model ${ }^{14,15}$ configured on a $1 / 10^{\circ}$ horizontal resolution grid with 54 vertical levels and forced with observed winds from the NCEP/NCAR reanalysis, with velocity data available at three-day temporal resolution. Figure 1 shows the mean speed of the surface flow (in red) and the mean direction of the surface flow (arrows). The means are computed as an average over time at fixed spatial points and, thus, represent Eulerian information as opposed to the semiLagrangian analysis carried out in the rest of this paper.

Our computational domain $X \subset \bar{X}$ consists of a twodimensional horizontal slice of the ocean at a depth of $5 \mathrm{~m}$ over the region extending from $75^{\circ} \mathrm{S}$ to $75^{\circ} \mathrm{N}$. Forward orbits of $X$ may permanently leave $X$ via beaching or being frozen into the Arctic or Antarctic, so $X \subset \bar{T}(X)$. We form $T:=\left.\bar{T}\right|_{X}: X \rightarrow X$, the restriction of $\bar{T}$ to $X$, and refer to $(X, T)$ as an open dynamical system, in contrast to the closed dynamical system $(\bar{X}, \bar{T})$.

In order to study the ocean's connectivity, we first form a spatial discretization of the dynamics, using a method known as Ulam's method ${ }^{16}$ see also Refs. 17 and 18 . We first grid the space $X$ into boxes $\left\{B_{i}\right\}_{i=1}^{N}$. We use $2^{\circ} \times 2^{\circ}$ boxes; discarding boxes $B_{i} \not \subset X$ leaves us with $N=10235$ boxes. Let $\mathcal{I}:=\{1, \ldots, N\}$, write $X_{N}:=\left\{B_{i}: i \in \mathcal{I}\right\}$ and define the collection of all sets that are unions of boxes in $X_{N}$ by $\mathbf{B}_{N}$. The proportion of mass in $B_{i}$ mapped to $B_{j}$ under one application of $T$ is equal to

$$
P_{i j}:=\frac{\operatorname{area}\left(B_{i} \cap T^{-1}\left(B_{j}\right)\right)}{\operatorname{area}\left(B_{i}\right)}, \quad i, j \in \mathcal{I},
$$

where area is normalized over $X$. The transition matrix $P$ defines a Markov chain representation $P$ of the dynamics, with the entries $P_{i j}$ equal to the conditional transition probabilities between boxes. In practice, the entries of $P$ must be numerically approximated using ocean trajectory data. Within the OFES vector fields, virtual particles are advected with a 4th-order Runge-Kutta method, using the connectivity modeling system v1.1 $1^{19}$ and a time step of $1 \mathrm{~h}$. Such a time step is sufficient to accurately track the particles in $0.1^{\circ}$ velocity fields like OFES. Because $T: X \rightarrow X$ is an open dynamical system, the matrix $P$ need not be stochastic, that is, $\sum_{j=1}^{N} P_{i j}$ may be strictly smaller than 1 for one or more $i \in \mathcal{I}$.

We initialize a set of $100 \mathrm{~N}$ particles on $X$ at $t=1 \mathrm{Jan}$. 2001 , with 100 particles placed on a $0.2^{\circ} \times 0.2^{\circ}$ lattice within each of the $N 2^{\circ} \times 2^{\circ}$ grid boxes (there are fewer particles in boxes that contain some land mass). We numerically estimate the entries of $P$ by calculating

$$
P_{i j}=\frac{\#\left\{x: x \in B_{i} \text { and } T(x) \in B_{j}\right\}}{\#\left\{x \in B_{i}\right\}} .
$$

In order to maintain a reasonably even sampling of points, we reinitialize the uniform distribution of particles every eight weeks to create six collections of consecutive eightweek trajectories and six transition matrices $P^{(1)}, P^{(2)}$, $\ldots, P^{(6)}$, as in Ref. 4. We then form $P=P^{(1)} \cdot P^{(2)} \cdot \ldots \cdot P^{(6)}$. The reinitialization in each box every eight weeks merely amounts to an eight-weekly uniform intra-box mixing and does not affect the overall dynamics of the Markov chain described by $P$.

Particles that exit $X$ at any point during the eight-week integration period are terminated. In total, we find that $0.5 \%$ of particles leave $X$ over the 48 -week period. Finally, in order to ensure that $P$ is not unduly influenced by the number of seed particles per box, we recalculated $P$ with 50 particles per box. The results presented in this paper were essentially unchanged when recalculated with reduced seeding.

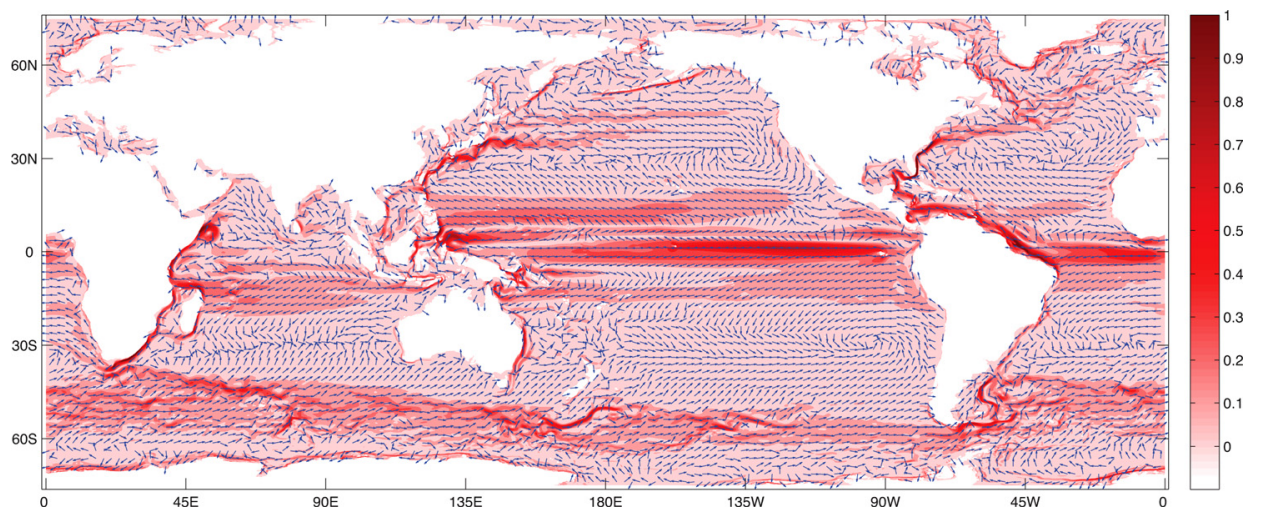

FIG. 1. Map of mean speed (red) in units of $\mathrm{m} / \mathrm{s}$ and direction (arrows) of the surface flow from the OFES model over 48 weeks. 


\section{FORWARD EVOLUTION OF TRAJECTORIES AND SURFACE UP- AND DOWN-WELLING}

We can use $P$ to visualize the forward evolution of a uniformly distributed set of points over the ocean's surface. Let $a$ be a vector with entries $a_{i}=\operatorname{area}\left(B_{i}\right), i \in \mathcal{I}$, and calculate $a^{(k)}:=a P^{k}$ for $k \in\{0,1,2, \ldots\}$. Figure 2 depicts fourth-root transformations of $a^{(2)}, a^{(10)}, a^{(100)}$, and $a^{(1000)}$, and we can observe a divergence of mass from the Equator and a convergence toward the centres of the subtropical gyres. Comparable results were obtained using a similar method in Refs. 3 and 4.

We can also calculate amounts of upwelling and downwelling over 48 weeks by imposing the restriction that the surface area of the ocean is preserved. Thus, if $a_{i}^{(1)}>a_{i}$, then the difference $a_{i}^{(1)}-a_{i}$ must have been pushed down below the ocean's surface (downwelled). Similarly, if $a_{i}>a_{i}^{(1)}$, then the difference $a_{i}-a_{i}^{(1)}$ must have emerged at the surface (upwelled). Ekman theory ${ }^{20}$ linearly relates the strength of the wind stress to the mass flux in the upper 10 to $100 \mathrm{~m}$ of the ocean. In our study, we assume that the thickness of the Ekman layer was a constant $50 \mathrm{~m}$ over the entire ocean. We, thus, think of the ocean surface area as a horizontal layer of $50 \mathrm{~m}$ depth. To calculate upwelling in the standard units of metres per day, we compute

$$
\frac{50 \max \left\{a_{i}-a_{i}^{(1)}, 0\right\}}{(7 \times 48) a_{i}},
$$

where the factor $7 \times 48$ accounts for the number of days in 48 weeks. To calculate downwelling, we use $a_{i}^{(1)}-a_{i}$ in place of $a_{i}-a_{i}^{(1)}$.

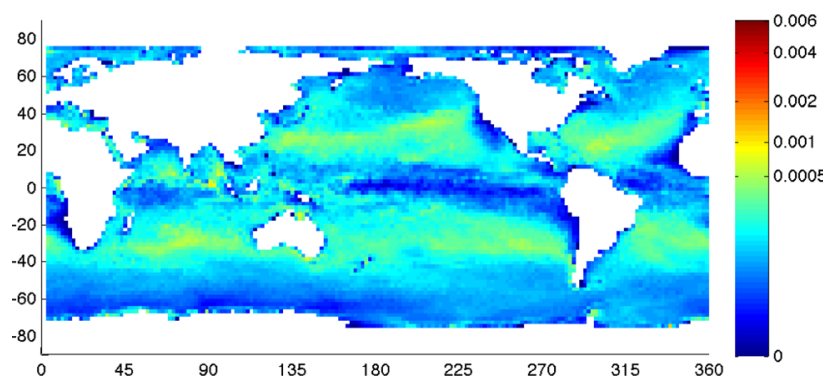

(a)

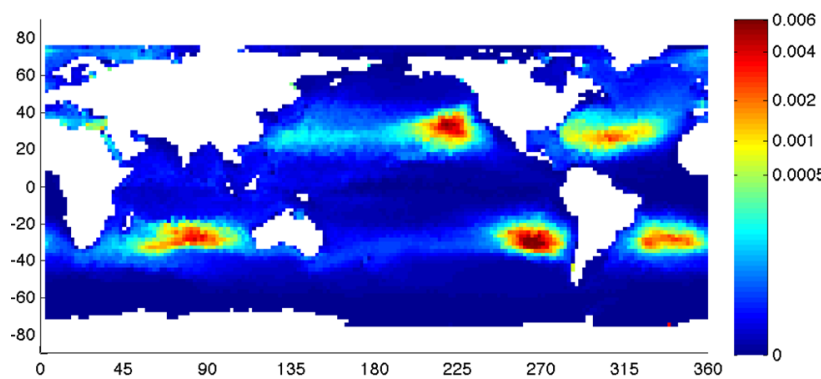

(c)
In Figure 3(a), we observe a large amount of upwelling occurring around the equator, the Western coastal regions of North and South America, and the Western coastal regions of Africa. Downwelling occurs in the North and South Pacific, the Indian, and the North and South Atlantic oceans (see Figure 3(b)), and is closely related to the regions where the garbage patches are located. ${ }^{4}$ The numerical values of both upwelling and downwelling are consistent with recent studies; see Figure 2(b), ${ }^{21}$ for example.

\section{ATTRACTING SETS, BASINS OF ATTRACTION, AND ABSORPTION PROBABILITIES}

In this section, we define some of the objects that we will use to analyze the connectivity of the surface ocean: attracting sets, basins of attraction, and absorption probabilities.

\section{A. Dynamical systems and attracting sets}

We will use a definition of attracting sets and basins of attraction based on Milnor. $^{22}$

Definition 1: Let $A^{c}:=X \backslash A$. A set $A$ for which area $\left(A \cap T^{-1}\left(A^{c}\right)\right)=0$ is called forward invariant. A forward invariant set $A$ for which area $\left(A^{c} \cap T^{-1}(A)\right)>0$ is called attracting. The basin of attraction $D_{A} \subset X$ for an attracting set $A$ is defined as the set of points whose forward orbits tend toward $A$.

$$
D_{A}:=\left\{x \in X: d\left(T^{k}(x), A\right) \rightarrow 0 \text { as } k \rightarrow \infty\right\}
$$

where $d(x, A)=\inf \{\operatorname{dist}(x, y): y \in A\}$.

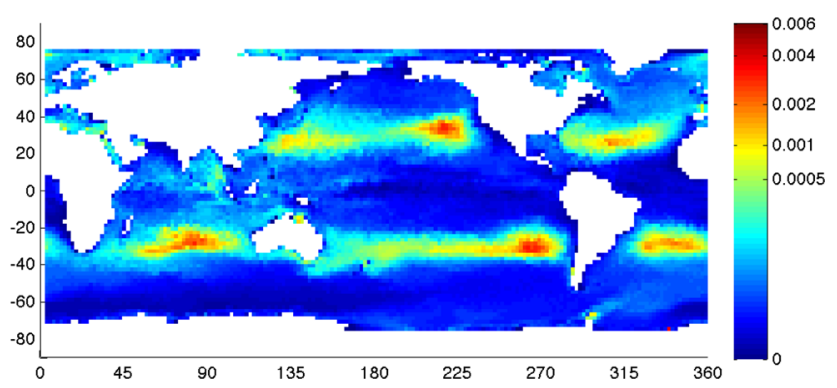

(b)

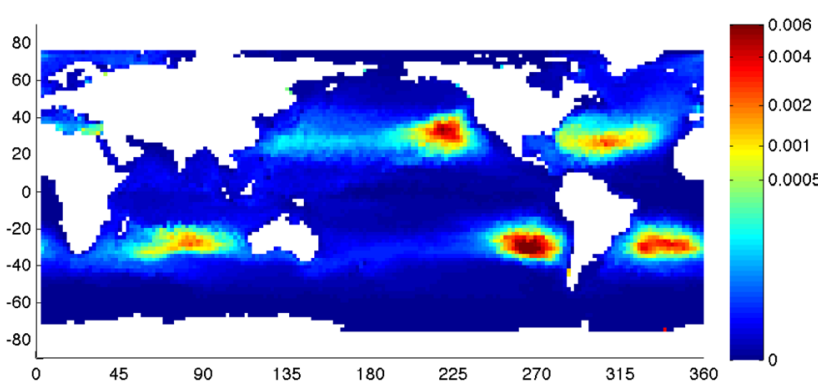

(d)

FIG. 2. Maps of evolution of a uniform density under the action of $P$. Densities are subjected to a fourth-root transformation. The color axes represent $a^{(k)}$, the horizontal and vertical axes represent the longitudinal and latitudinal coordinates, respectively. 


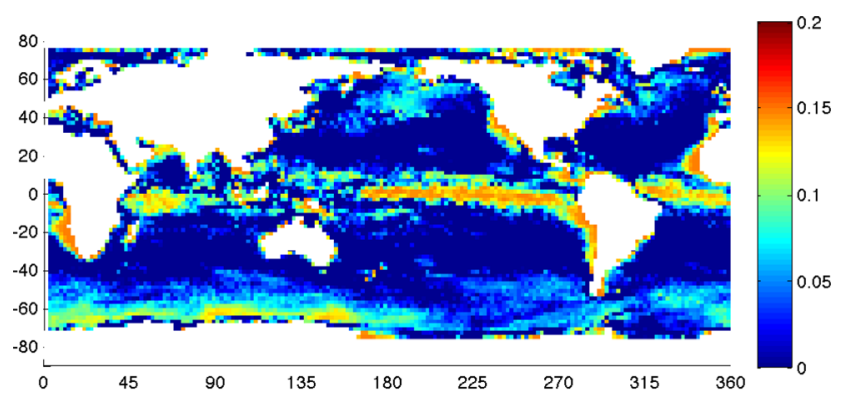

(a)

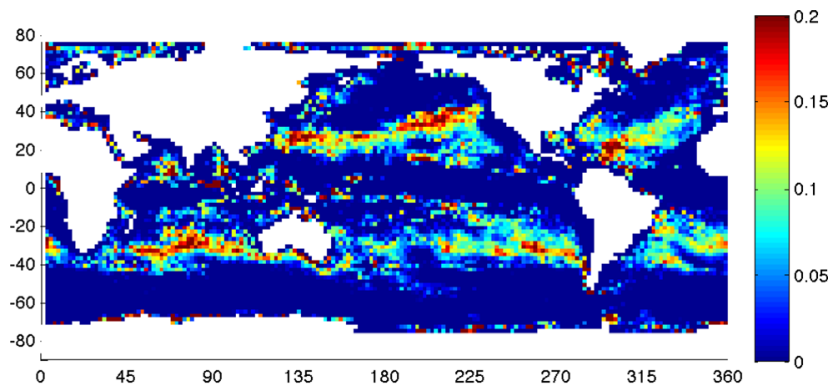

(b)

FIG. 3. Maps of average upwelling and downwelling rates. (a) Average rate of upwelling over 48 weeks (in m/day). (b) Average rate of downwelling over 48 weeks (in $\mathrm{m} /$ day).

\section{B. Markov chains and absorption probabilities}

In Sec. II, we defined a Markov chain representation of the dynamics, with the conditional transition probabilities defined by (1). We will now use this representation to relate attracting sets for dynamical systems to absorbing classes for Markov chains.

Definition 2: We refer to the set of states $S_{k} \subset \mathcal{I}$ as an absorbing closed communicating class if one has:

For each pair $i, j \in S_{k}$, there exists an $m>0$ such that $P_{i j}^{m}>0$.

(iii) $\quad P_{i j}>0$ for at least one $i \notin S_{k}, j \in S_{k}$.

Property (i) says that each class is communicating (there is a positive probability of moving between any pairs of states in the class in a finite number of steps); property (ii) says that each class is closed (there is a zero probability of moving out the class to another state elsewhere in our computational domain $X$; we do not, however, restrict movement from states in the class to states outside of $X$, and allow the possibility that $\sum_{j} P_{i j}<1$ for $i \in S_{k}$ ); property (iii) says that each class is absorbing (there is a positive probability of external states moving into the class in $m$ steps). The notation $S_{k}$ allows for the possibility that there are $K$ absorbing closed communicating classes, each identified by an index number $k \in\{1, \ldots, K\}$. We can connect Definitions 1 and 2 according to the following simple Lemma:

Lemma 1: If $S_{k}$ is an absorbing closed communicating class for the Markov chain defined by $P$ in (1), then $A_{k}:=\cup_{i \in S_{k}} B_{i}$ is an attracting set for the open dynamical system $T: X \rightarrow X$.

Proof: See Appendix A.

Markov chain theory also allows us to compute the probability, denoted $h_{k, i}$, of eventually hitting an absorbing closed communicating class $S_{k}$, conditioned on beginning in state $i \notin S_{k}$. As each class $S_{k}, k=1, \ldots, K$ is closed, clearly $h_{k, i}=0$ for $i \in \cup_{j=1, j \neq k}^{K} S_{j}$, so we are only interested in $h_{k, i}$ for $i \notin \cup_{j=1}^{K} S_{j}$.

The dynamical systems interpretation of $h_{k, i}$ is the proportion of particles beginning in $B_{i} \not \subset A_{k}$ that eventually hit the attracting set $A_{k}:=\cup_{j \in S_{k}} B_{j}$. A result adapted from Ref. 23 and used by Ref. 24 in a dynamical systems context provides a method for exactly computing the vector of absorption probabilities $h_{k}=\left(h_{k, i}\right)$. We extend the method of Ref. 24 in two ways: first, we allow for the possibility that particles in the attracting sets may leak out of the domain, and second, we handle attracting sets consisting of multiple states. We first define $\mathcal{I}_{S}:=\cup_{k} S_{k}, \mathcal{I}_{T}:=\mathcal{I} \backslash \mathcal{I}_{S}$, and $\left\{\hat{P}_{k}\right\}_{k \in\{1, \ldots, K\}}$ by

$$
\hat{P}_{k}=\left[\begin{array}{cc}
\Pi_{k} P_{k} & 0 \\
R_{k} & Q_{k}
\end{array}\right],
$$

where $P_{k, i j}=P_{i j}$ for $i, j \in S_{k}, \Pi_{k}$ is a diagonal matrix of size $\left|S_{k}\right|$ with $\Pi_{k, i i}=1 / \sum_{j \in S_{k}} P_{i j}$ for $i \in S_{k}, R_{k, i j}=P_{i j}$ for $j \in S_{k}, i \in \mathcal{I}_{T}$, and $Q_{k, i j}=P_{i j}$ for $i, j \in \mathcal{I}_{T}$.

Theorem 1: The vector of absorption probabilities $h_{k}=$ $\left(h_{k, i}\right)_{i \in S_{k} \cup \mathcal{I}_{T}}$ into an absorbing closed communicating class $S_{k}$ is the minimal nonnegative solution to

$$
\hat{P}_{k} g=g,
$$

where $g$ is a vector constrained to have $g_{i}=1$ for $i \in S_{k}$.

Proof: See Appendix B.

The following algorithm summarizes the steps necessary to define $P$ and calculate the attracting sets.

Algorithm 1:

1. Partition the computational domain $X$ into connected sets $\left\{B_{1}, B_{2}, \ldots, B_{N}\right\}$.

2. Construct the transition matrix $P$ corresponding to the open system, following (2).

3. Determine the absorbing closed communicating classes of $P$. The communicating classes may be easily and quickly computed using, e.g., Tarjan's algorithm. ${ }^{25}$

Applying Algorithm 1 to the global ocean's surface in the OFES model, we identify 10 absorbing closed communicating classes (or sinks): 5 in the North Pacific regions, 1 in the North Atlantic, 2 North of Alaska, 1 off the coast of Peru, and 1 in the Southern Ocean. Each of these absorbing classes is comprised of 1 or 2 boxes from which no trajectories leave and into which some trajectories enter from outside. Each sink lies adjacent to a coastline, and it is likely that the precise locations and number of these sinks are artefacts of the OFES model and the box discretisation; we do not place any physical significance on them and refer to them as nonphysical absorbing classes. For this reason, we do not report on the corresponding absorption probabilities, although to compute the vectors $h_{k}$ by solving (6) is simple to implement in, e.g., MATLAB using the backslash command. Nevertheless, for our eigenvector methods described 
in Sec. V, it is important to know about these sinks as they will appear as eigenvectors, which we will discard due to their nonphysicality. It is interesting to note that none of the garbage patches represent or contain sinks. As described in Ref. 4, the garbage patches are "leaky attractors."

\section{EIGENVECTOR METHODS}

In this section, we present our main approach for analysing the global dynamics of the OFES model. Our strategy is to compute eigenvectors of the matrix $P$ corresponding to real eigenvalues of $P$ close to 1 .

\section{A. Left eigenvectors of $P$}

To motivate our approach, let us first consider an idealised situation where the transition matrix $P$ generated from the OFES model contains $M$ small nonphysical absorbing classes and 5 garbage patches, each of which is also an absorbing closed communicating classes, and further that the basins of attraction are pairwise disjoint. In this idealised situation, we also assume that there is no loss of trajectories through beaching or being frozen in ice, so the matrix $P$ is row-stochastic. In such a situation, the leading eigenvalue of $P$ is 1 , this eigenvalue has multiplicity $M+5$, and one may find a basis of eigenvectors supported on the (disjoint) box collections comprising the basins of attraction. The matrix $P$ has a block diagonal structure consisting of $M$ diagonal unitsized blocks with entries 1 , and 5 further larger blocks corresponding to the 5 garbage patches.

Suppose now that we perturb $P$ continuously; by classical matrix perturbation theory (Theorem II.5.1 of Ref. 26), one knows that the $M+5$ copies of the eigenvalue 1 will move continuously as a group, possibly becoming $M+5$ distinct eigenvalues nearby 1 . Further, under such a perturbation, the span of the $M+5$-dimensional eigenspace corresponding to the eigenvalue 1 also moves continuously to a nearby group of eigenspaces of the perturbed $P$ of total dimension $M+5$. Arguing in this way, we expect the (signed) supports of the top $M+5$ eigenvectors of the perturbed $P$ to reveal to us (through linear combinations) the supports of the unperturbed $P$. Precursors of these ideas are discussed ${ }^{27}$ in the simpler situation of a row-stochastic, reversible $P$, and methods are put forward ${ }^{28}$ to find approximately orthogonal bases in the same setting (see also Ref. 29 for related results).

The perturbation we have in mind here is the real OFES model as opposed to the idealised model sketched above. The real OFES model has a small loss of trajectories (due to beaching and freezing), leading to a substochastic $P$. The real OFES model does not have exactly disjoint basins of attraction at the discrete grid level. Finally, the garbage patches are not infinite-time absorbing classes, but very long-term absorbing classes (many thousands of years). Nevertheless, we show that this perturbation approach works very well for the OFES model.

On the basis of the above argument, our hope is that the span of the leading eigenvectors will reproduce a linear combination of the 10 nonphysical absorbing classes, as well as the garbage patches distributions in Figure 2(d), which represent conditional transient behaviour that very slowly enters one of the absorbing classes. For $P$ constructed from the OFES model, we have a complicated mix of eigenvalues close to 1 . Some eigenvalues correspond to eigenvectors associated with absorption to absorbing classes comprised of one or two boxes (with possible loss of trajectories, so that the eigenvalue may be slightly less than 1) and some to exchange between large collections of boxes corresponding to garbage patches. The former are the nonphysical absorbing classes, and we are primarily interested in the latter larger collections. The leading eigenvalues of $P$ are listed in Table I.

In practice, we visualise the eigenvectors to determine their supports. Figure 4 shows left eigenvectors of $P$ close to 1 , whose eigenvalues correspond to small exchange between larger collections of boxes. The eigenvectors clearly highlight five ocean garbage patches, present in the North and South Pacific, Indian, and North and South Atlantic Oceans, ${ }^{1-4}$ consistent with Figure 2(d). Dynamically, this makes sense because there is likely to be only very little exchange between the (attracting) garbage patches.

In this particular case study, the top four eigenvectors and the eighth eigenvector (not shown) highlighted combinations of boxes related to the (possibly leaky) nonphysical absorbing classes. Further down the spectrum at positions 5, $6,7,9$, the eigenvectors describe the slow-exchange dynamics between the garbage patches (Figure 4). For the eigenvectors $5,6,7,9$, the eigenvalues quantify the geometric rates at which the (signed) densities shown in Figure 4 converge to equilibrium. The eigenvectors themselves are (scalar multiples of) signed densities, representing the slowdecaying modes. Those eigenvectors with eigenvalues closest to 1 are the most important, as they are the slowest to decay and are the most long-lived transient modes. Some of these eigenvectors highlight the patches along with some of the nonphysical absorbing classes, for example, Figure 4(a) shows a small highlighted region on the southwest coast of South America, in addition to two garbage patches in the South Pacific and South Atlantic.

TABLE I. Top 15 eigenvalues for $P$ and $\hat{P}$.

\begin{tabular}{lcc}
\hline \hline$\lambda$ & $P$ & $\hat{P}$ \\
\hline$\lambda_{1}$ & 1.0000 & 1.0000 \\
$\lambda_{2}$ & 1.0000 & 1.0000 \\
$\lambda_{3}$ & 1.0000 & 1.0000 \\
$\lambda_{4}$ & 1.0000 & 1.0000 \\
$\lambda_{5}$ & 0.9999 & 1.0000 \\
$\lambda_{6}$ & 0.9999 & 0.9999 \\
$\lambda_{7}$ & 0.9996 & 0.9999 \\
$\lambda_{8}$ & 0.9991 & 0.9996 \\
$\lambda_{9}$ & 0.9975 & 0.9991 \\
$\lambda_{10}$ & 0.9913 & 0.9975 \\
$\lambda_{11}$ & 0.9852 & 0.9913 \\
$\lambda_{12}$ & 0.9838 & 0.9852 \\
$\lambda_{13}$ & 0.9826 & 0.9838 \\
$\lambda_{14}$ & 0.9680 & 0.9826 \\
$\lambda_{15}$ & 0.9645 & 0.9680 \\
\hline \hline
\end{tabular}




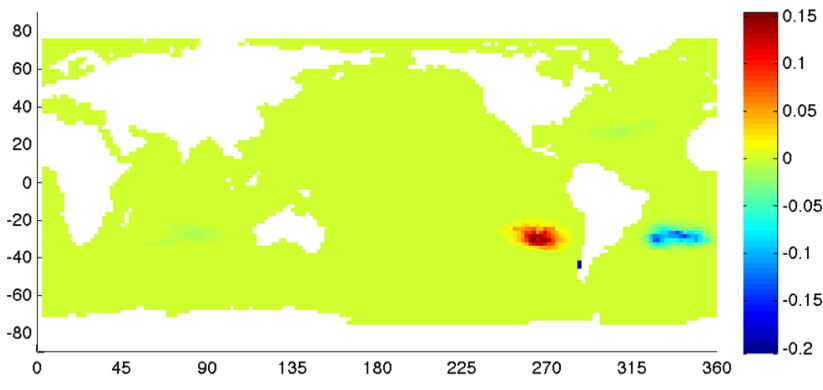

(a)

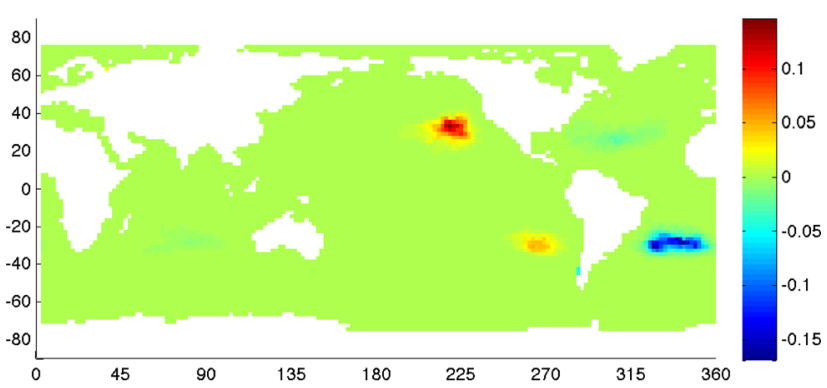

(b)

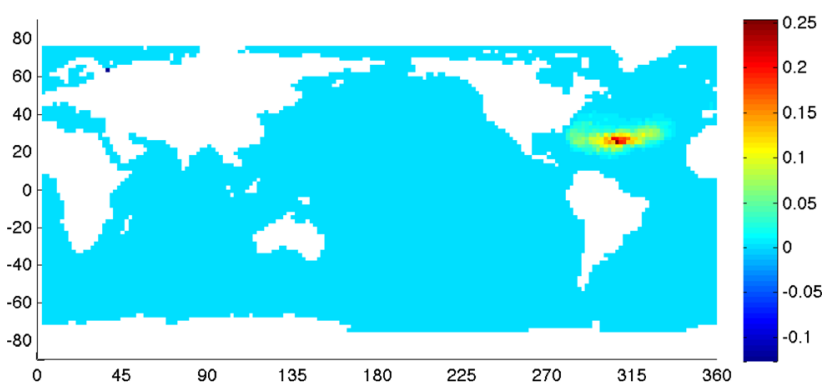

(c)

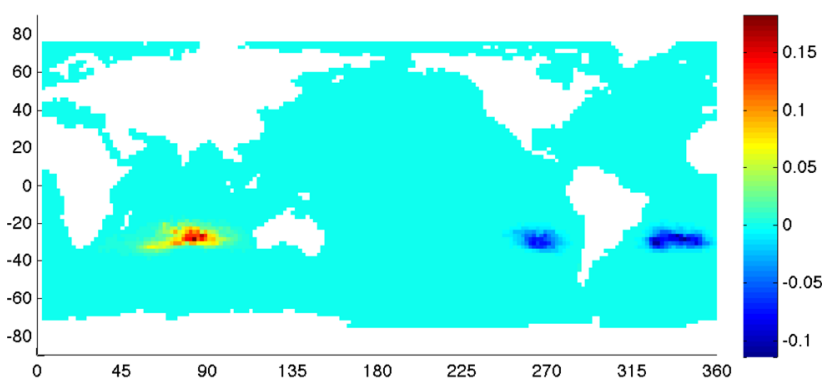

(d)

FIG. 4. Maps of selected left eigenvectors of $P$ showing the locations of the five great ocean garbage patches.

\section{B. Right eigenvectors of $\boldsymbol{P}$}

Let us now turn to the right eigenvectors of $P$. Clearly we have the same complex mix of eigenvalues as discussed above. Under left multiplication, the matrix $P^{\top}$ is the matrix representation of the dual dynamical action of $P$; see Lemma $5 .^{30}$ Thus, the right eigenvectors $v$ of $P$ associated with real eigenvalues $\lambda \neq 1$ close to 1 have similar properties to the left eigenvectors, with two important differences: they capture backward-time dynamics and rather than spanning a space that looks approximately like the long-term mass distribution $p$ (in the OFES model, this distribution is concentrated in the nonphysical absorbing classes and in the garbage patches) restricted to subregions, they span a space that looks approximately like 1 restricted to subregions.

Moreover, by comparison with Theorem 1, one can interpret the indicated regions as basins of attraction. Let us consider again the idealised situation of Sec. V A: The transition matrix $P$ has $M$ nonphysical absorbing classes and 5 garbage patches, each of which is an absorbing closed communicating class, with pairwise disjoint basins of attraction. Further, there is no loss of trajectories so that $P$ is stochastic. There will be $M+5$ eigenvalues with eigenvalue 1 . What do the right eigenvectors look like? A particular basis is provided by Theorem 1 . Consider $k \in\{1, \ldots, M+5\}$. Applying Theorem 1 (note $\rho_{k}$ is a vector of $1 \mathrm{~s}$ ), we see the entries of the corresponding right eigenvector $v_{k, i}$ are

$$
v_{k, i}= \begin{cases}1, & i \in S_{k} \\ h_{k, i}, & i \text { is in the basin of attraction of } S_{k} \\ 0, & i \text { is not in the basin of attraction of } S_{k}\end{cases}
$$

Since the $S_{k}$ are pairwise disjoint and the basins are pairwise disjoint, the vectors $\left\{v_{k}\right\}_{k=1}^{M+5}$ have disjoint support, with $v_{k}$ supported only on $S_{k}$ and its basin of attraction.

If we now perturb $P$ to obtain the real $P$ obtained from the OFES model, again appealing to classical matrix perturbation theory, we expect to obtain $M+5$ eigenvalues nearby 1. Moreover, the level structure of the idealised $v_{k}$ indicated in (7), which separates the different basins of attraction, will persist in the (now signed) values of the $v_{k}$. Thus, the level structure of the right eigenvectors $\mathrm{v}_{\mathrm{k}}$ close to 1 should separate the basins of attraction for distinct absorbing closed communicating classes.

In Figure 5, we show four right eigenvectors of $P$, corresponding to the four left eigenvectors in Figure 4. The sets shown in deep red and light blue in the southern hemisphere in Figure 5(a) correspond to the South Pacific and South Atlantic garbage patches, respectively, in Figure 4(a). The deep red region in Figure 5(b) corresponds to the North Pacific garbage patch; the set shown in orange in the North Atlantic in Figure 5(c) corresponds to the North Atlantic garbage patch, and the deep red region in Figure 5(d) corresponds to the Indian Ocean garbage patch; compare with Figures 4(b)-4(d), respectively.

We can form an almost full partition of the surface ocean by combining the information in Figure 5; see Figure 6. For example, the North Pacific component in Figure 6 comprises boxes such that the value of $v_{P, 6}$ is above 0.005 . We can then calculate the condition transition probabilities between the regions identified in the partition in Figure 6, over a 48-week duration in time. Table II shows the result of this calculation, with I is the Indian, NP is the North Pacific, SP is the South Pacific, NA is the North Atlantic, SA is the South Atlantic, and $\mathrm{R}$ is the remainder (unassigned or the extreme north and south). The inter-region percentages along the diagonal of the array are all near $100 \%$, which is consistent with the interpretation of the regions as basins of attraction of a garbage patch. The main losses (above 2\%) are from the North Pacific to the South Pacific (3.1\%) and from 
TABLE II. Forward-time conditional transition percentages between the five surface ocean regions (from row to column) shown in Figure 6, associated to domains of attraction for each of the five major garbage patches.

\begin{tabular}{lcccccc}
\hline \hline & $\mathrm{I}$ & $\mathrm{NP}$ & $\mathrm{SP}$ & $\mathrm{NA}$ & $\mathrm{SA}$ & $\mathrm{R}$ \\
\hline $\mathrm{I}$ & 95.43 & 0.56 & 1.39 & 0 & 1.92 & 0.54 \\
$\mathrm{NP}$ & 1.50 & 94.89 & 3.10 & 0 & 0 & 0.42 \\
SP & 1.31 & 1.10 & 95.83 & 0 & 0.46 & 1.25 \\
NA & 0 & 0 & 0 & 97.14 & 0.98 & 1.44 \\
SA & 6.56 & 0 & 0.34 & 0.03 & 91.62 & 1.44 \\
\hline \hline
\end{tabular}

the South Atlantic to the Indian (6.56\%). Note that Table II is non-symmetric, which is consistent with the lack of timesymmetry of the surface ocean dynamics.

Note that the flow direction indicated by the sweep of the sets indicated in Figure 5 is consistent with a backwardtime ocean flow. This is as expected, since right eigenvectors of $P$ correspond to backward-time dynamics. Interestingly, these basins of attraction do not completely overlap with the geographical notion of the different ocean basins. In particular, in the Southern Hemisphere, the attracting regions "spill over" westward: the South Atlantic region in Figure 5(d) extends through Drake Passage and well into the Pacific sector of the Southern Ocean. The strong Antarctic Circumpolar Current (ACC) apparently skews the water mass distributions in the Southern Ocean. The equatorial region is also interesting from an oceanographic point of view. The northern hemisphere attracting regions extend further south in the Atlantic than in the Pacific (Figure 5(a)). This is very likely due to the presence of the North Brazil Current, ${ }^{31}$ which breaks the hemispheric symmetry in the Atlantic Ocean and has no counterpart in the tropical Pacific Ocean.

\section{Left eigenvectors of $\hat{P}$ : OFES model}

Finally, we wish to create a forward-time partition of the ocean surface "analogous" to the backward-time partition shown in Figure 6. By "analogous," we mean that we would like to have a complete partition of the ocean that takes the focus away from the dominant attracting regions (the sinks and the garbage patches), which were so readily highlighted by the left eigenvectors of $P$.

There are two equivalent ways of doing this. First, we can take a left eigenvector $u_{P, k}, k=5,6,7,8$ of $P$ and divide element-wise by $a^{(1000)}$ to produce $\left(v_{\hat{P}, k}\right)_{i}=\left(u_{P, k}\right)_{i} /$ $\left(a^{(1000)}\right)_{i}$; the notation for $v_{\hat{P}, k}$ will become clear shortly. Recall that $a^{(1000)}$ is the pushforward of a uniform measure on the ocean over 1000 cycles (depicted in the final panel of Figure 2). The vectors $v_{\hat{P}, k}$ describe forward-time transient behaviour, "modulo" the sinks and garbage patches as they appear from the 1000-year evolution because the values of $u_{P, k}$ are proportionally diminished around these features.

Second, we can form a matrix that is akin to a timereversal of the transition matrix $P$. The standard way to do this is to use the invariant measure $p$ (see, e.g., Ref. 32 and in the dynamical systems setting ${ }^{33}$ ). The problem with the OFES model is that there is not a single $p$, but at least 12 (conditional) invariant densities, corresponding to the distinct nonphysical absorbing classes. Rather than consider the

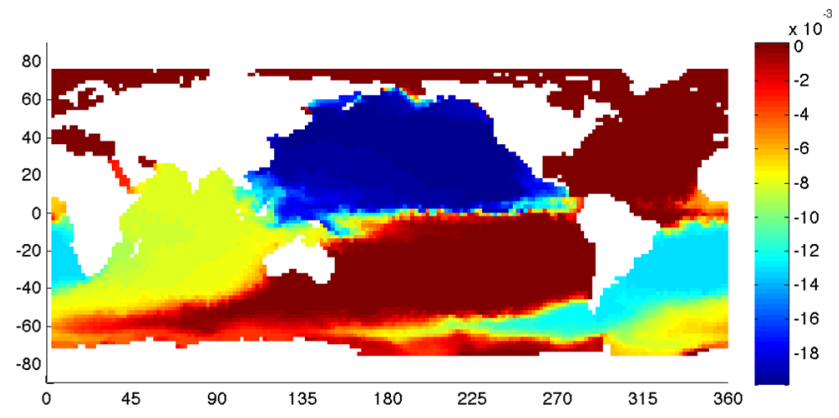

(a)

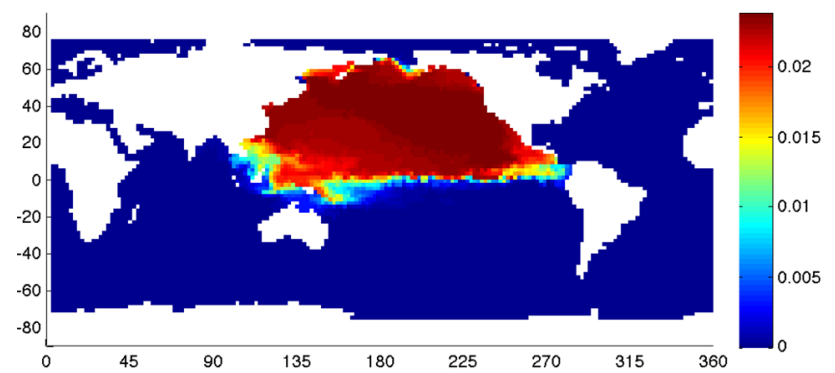

(b)

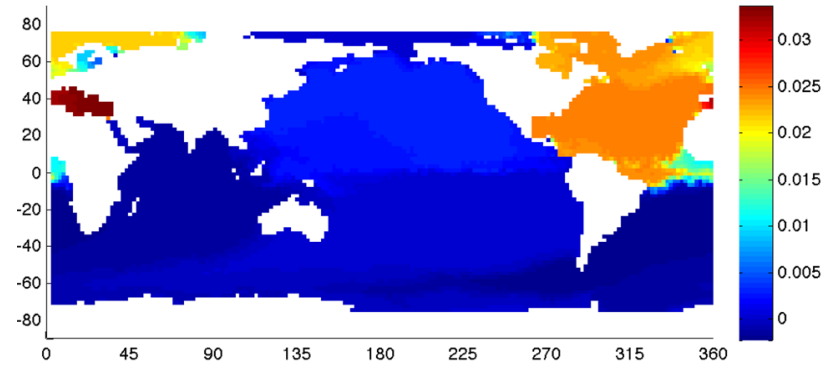

(c)

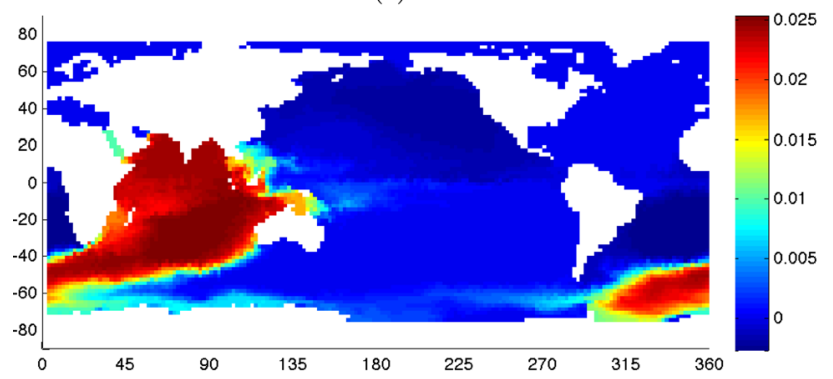

(d)

FIG. 5. Maps of right eigenvectors $\left\{v_{P, r}\right\}$ of $P$.

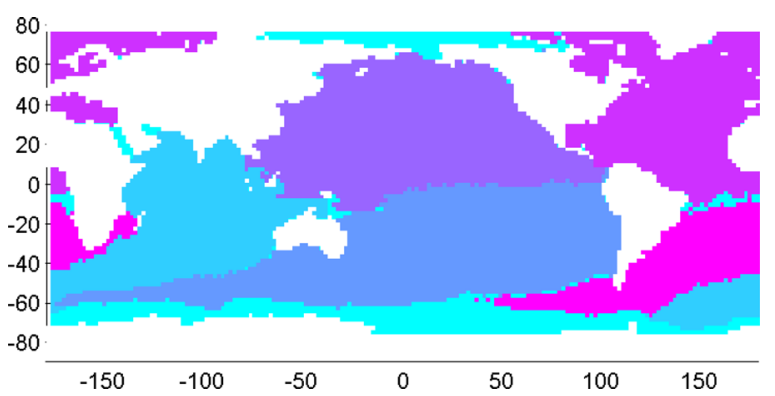

FIG. 6. Map of a partition of the surface ocean into domains of attraction associated to each of the five major garbage patches, based on backward time evolution. 
(multiple) infinite futures of surface particles, we again use the 1000-year future, namely, $\eta:=a^{(1000)}$ as a proxy for $p$. The matrix $\hat{P}_{i j}:=\eta_{j} P_{j i} / \eta_{i}$ represents an approximate backward-time dynamics under left multiplication. The vector $\eta$ represents an approximate invariant measure of the dynamics of $\hat{P}$, since $\sum_{i} \eta_{i} \hat{P}_{i j}=\eta_{j} \sum_{i} P_{j i}$, and $\sum_{i} P_{j i}$ is approximately a vector of $1 \mathrm{~s}$ (it is not exactly $\mathbf{1}$ because of the tracer leakage).

We now compute the right eigenvectors $v_{\hat{P}}$ of $\hat{P}_{i j}$, which contain information about the almost-invariant regions for the flow in forward-time, and importantly span a space that looks approximately like $\mathbf{1}$ restricted to subregions. The following computation shows that these two methods of constructing $v_{\hat{P}}$ coincide. Using the second characterisation, let $v_{\hat{P}}$ be a right eigenvector of $\hat{P}$ with eigenvalue $\lambda$. Then

$$
\lambda\left(v_{\hat{P}}\right)_{i}=\sum_{j=1}^{n} \hat{P}_{i j}\left(v_{\hat{P}}\right)_{j}=\sum_{j=1}^{n} \frac{\eta_{j} P_{j i}}{\eta_{i}}\left(v_{\hat{P}}\right)_{j} .
$$

Thus, $\lambda\left[\eta_{i}\left(v_{\hat{P}}\right)_{i}\right]=\sum_{j=1}^{n}\left[\eta_{j}\left(v_{\hat{P}}\right)_{j}\right] P_{j i}$, so $\eta * v_{\hat{P}}$ is a left eigenvector $u_{P}$ of $P$ with eigenvalue $\lambda$, where $*$ denotes componentwise multiplication; rearranging, $\left(v_{\hat{P}}\right)_{i}=\left(u_{P}\right)_{i} / \eta_{i}$ as in our first construction. The eigenvalues corresponding to $\hat{P}$ are given in Table I. The eigenvalues of $P$ and $\hat{P}$ are very close as expected, since $\hat{P}$ is a similarity transformation of $P$.

In Figure 7, we present the four right eigenvectors of $\hat{P}$ that correspond to the four eigenvectors shown in Figures 4 and 5. In Figure 7(a), one can identify a yellow patch extending outwards from South America to the east, and a deep red triangular patch extending west, corresponding to the South Atlantic and South Pacific garbage patches. In Figure 7(b), several colored regions are shown, each corresponding to a distinct garbage patch. In Figures 7(c) and 7(d), one sees the deep red region in the North Atlantic and the deep red region extending out from the Indian Ocean to the east, past southern Australia and South America.

As in Sec. V B, we can combine the information in the eigenvectors to form an approximate partition of the surface ocean, shown in Figure 8. Because this partition is constructed from forward-time eigenvectors, it can be thought of as a partition into the medium to long-term forward-time transient structure of the ocean, modulo the absorbing features of sinks and gyres.

Table III shows the conditional transition probabilities over a 48 week duration between the five ocean domains in Figure 8.The values on the diagonal of this table are not as large as those in Table II because we are not carrying out the division by $a^{(1000)}$ and the numbers are based on a single annual cycle, rather than hundreds of cycles.

Figure 7 shows some interesting features of inter-ocean connections. The main structures visible in Figure 7(b) are in the southeast Pacific, in an area (yellow) roughly overlapping with the cold tongue there ${ }^{34}$ but also including a bit of the South Atlantic around Drake Passage. There is a structure in Figure 7(d) (dark blue) in the South Atlantic, that interestingly, excludes the Benguela upwelling, ${ }^{35}$ which, according to Figure 7(d), is more connected to a large structure (red) encompassing all of the Indian Ocean, the southwestern part

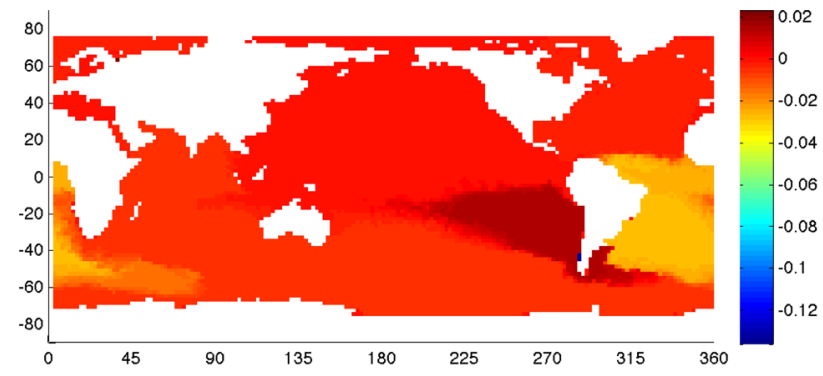

(a)

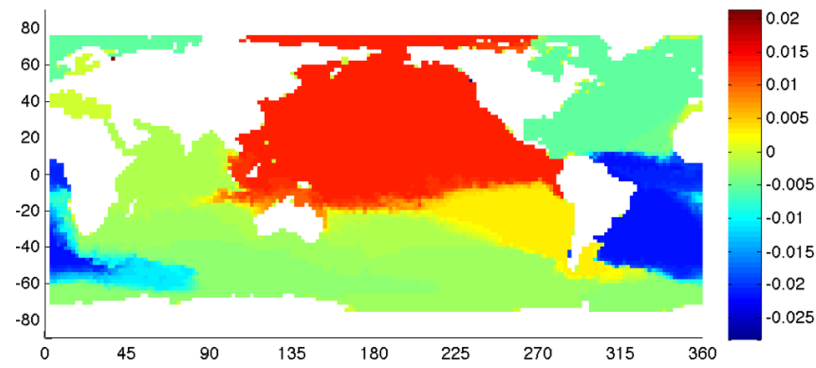

(b)

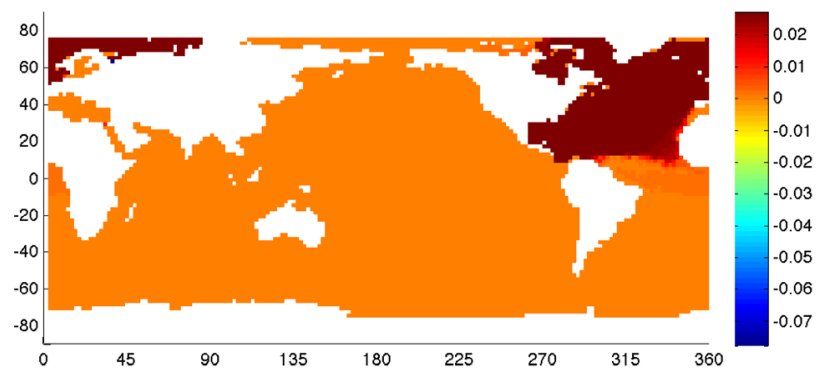

(c)

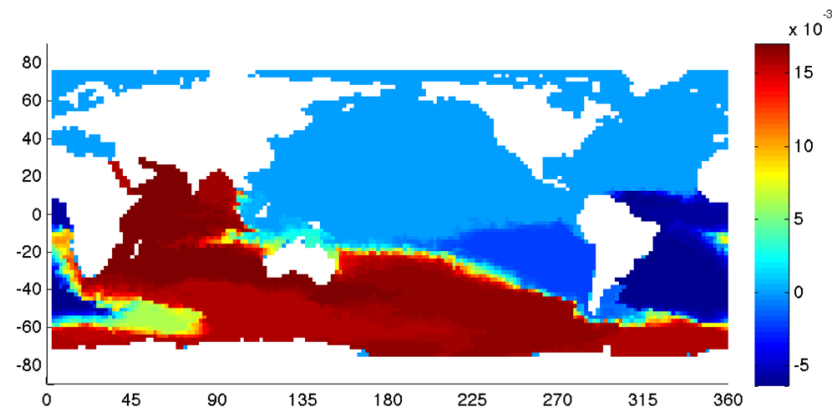

(d)

FIG. 7. Maps of right eigenvectors $\left\{v_{\hat{P}, r}\right\}$ of $\hat{P}$.

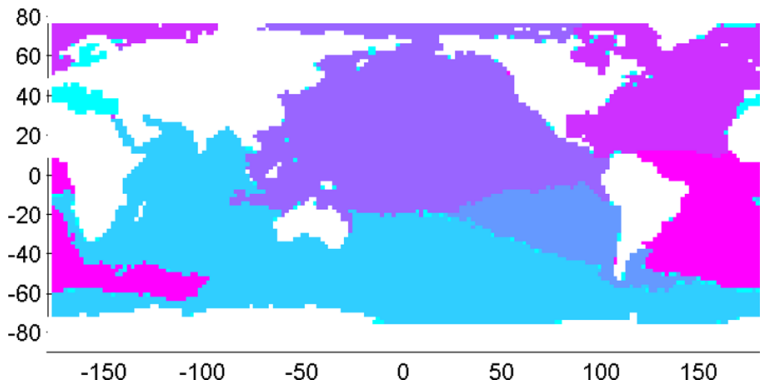

FIG. 8. Map of a partition of the surface ocean based on forward-time evolution. 
TABLE III. Forward-time conditional transition percentages between the five surface ocean regions (from row to column) shown in Figure 8.

\begin{tabular}{lcccccc}
\hline \hline & $\mathrm{I}$ & $\mathrm{NP}$ & $\mathrm{SP}$ & $\mathrm{NA}$ & $\mathrm{SA}$ & $\mathrm{R}$ \\
\hline I & 90.12 & 0.51 & 3.94 & 0 & 3.67 & 0.71 \\
NP & 3.78 & 92.68 & 2.78 & 0.09 & 0.01 & 0.32 \\
SP & 3.50 & 1.23 & 86.36 & 0 & 8.51 & 0.39 \\
NA & 0 & 0.03 & 0 & 99.00 & 0.51 & 0.02 \\
SA & 13.05 & 0 & 0 & 8.66 & 77.82 & 0.34 \\
\hline \hline
\end{tabular}

of the Pacific and nearly all of the Southern Ocean. In the tropics, the Indonesian through flow (the phenomenon by which water from the Pacific flows into the Indian Ocean via the Indonesian Archipelago ${ }^{36}$ ) is also visible in both Figures 7(b) (red) and 7(d) (light blue).

\section{CONCLUSION}

Ulam's method, now a staple tool in many dynamical systems applications, enables one to use Markov chain ideas to analyse dynamical systems. Employing this method, we built a Markov representation of the dynamics of the global surface ocean. We were then able to compute the evolution of densities over many centuries, calculate surface upwelling and downwelling, and identify attracting regions using strongly connected components, exploiting a connection between attracting sets for dynamical systems and absorbing closed communicating classes of Markov chains. Finally, we were able to interpret the left and right eigenvectors of the Markov chain transition matrix as almost-invariant sets and basins of attraction, respectively. We used these eigenvector techniques as a powerful method of identifying garbage patch locations and mapping their basins of attraction. We thus dynamically decomposed the global ocean surface into weakly interacting parts in both forward and backward time.

Decompositions such as these one can form the basis of a dynamical geography of the ocean surface, where the boundaries between basins are determined from the circulation itself, rather than from arbitrary geographical demarkations. One of the results that comes out of this decomposition, for instance, is that the Atlantic and Pacific Oceans are split into Northern and Southern parts at roughly the Equator, but that the Indian Ocean is one entity from $30^{\circ} \mathrm{S}$ to $30^{\circ} \mathrm{N}$. Maps of these kinds of features in the ocean's dynamical geography allow us to better understand how the different ocean basins are connected.

\section{ACKNOWLEDGMENTS}

G.F. was supported by an ARC Future Fellowship. R.M.S. was supported by an Australian Postgraduate Award and the ARC Centre of Excellence for the Mathematics and Statistics of Complex Systems (MASCOS). E.v.S. was supported by the Australian Research Council via Grant No. DE130101336.

\section{APPENDIX A: PROOF OF LEMMA 1}

Only properties (ii) and (iii) of Definition 1 are required. Suppose $S_{k}$ is an absorbing closed communicating class for the Markov chain with conditional transition probabilities given by (1). Then, $P_{i j}=0$ for $i \in S_{k}, j \notin S_{k}$, which implies that area $\left(A \cap T^{-1} A_{j}\right)=0$ for all $j \notin S_{k}$, so $A$ is a forward invariant set. Also, $P_{i j}>0$ for $j \in S_{k}$ and at least one $i \notin S_{k}$, which implies that $\operatorname{area}\left(A_{i} \cap T^{-1}(A)\right)>0$ for at least one $i \notin S_{k}$, so $A$ is an attracting set according to Definition 1 .

\section{APPENDIX B: PROOF OF THEOREM 1}

The proof is an expanded version of the proof of Theorem 1.3.2 ${ }^{23}$ we present the proof here for completeness to demonstrate the application to the substochastic setting.

Let $\left(Z_{t}\right)_{t \in \mathbb{N}}$ be the substochastic Markov process over state space $\mathcal{I}$, with conditional transition probabilities $\mathbb{P}\left\{Z_{t+1}=j \mid Z_{t}=i\right\}=P_{i j}$ for $i, j \in \mathcal{I}$.

First, note that if $i \in S_{k}$, then $h_{k, i}=\mathbb{P}\left\{Z_{t+0} \in S_{k} \mid\right.$ $\left.Z_{t} \in S_{k}\right\}=1$. Next, we examine the case $i \in \mathcal{I}_{T}$. We have

$$
\begin{aligned}
h_{k, i}:= & \mathbb{P}\left\{Z_{t+r} \in S_{k}, \text { some } r \geq 1 \mid Z_{t}=i\right\}, \\
= & \mathbb{P}\left\{Z_{t+r} \in S_{k}, \text { some } r \geq 2, Z_{t+1} \in I_{T}, Z_{t}=i\right\} / p_{i} \\
& +\mathbb{P}\left\{Z_{t+1} \in S_{k} \mid Z_{t}=i\right\}, \\
= & \sum_{j \in I_{T}} \mathbb{P}\left\{Z_{t+r} \in S_{k}, \text { some } r \geq 2, Z_{t+1}=j, Z_{t}=i\right\} / p_{i} \\
& +\sum_{j \in S_{k}} P_{i j}, \\
= & \sum_{j \in I_{T}} \mathbb{P}\left\{Z_{t+r} \in S_{k}, \text { some } r \geq 2 \mid Z_{t+1}=j\right\} \\
& \cdot \mathbb{P}\left\{Z_{t+1}=j, Z_{t}=i\right\} / p_{i}+\sum_{j \in S_{k}} P_{i j}, \\
= & \sum_{j \in I_{T}} \mathbb{P}\left\{Z_{t+r} \in S_{k}, \text { some } r \geq 1 \mid Z_{t}=j\right\} \cdot P_{i j}+\sum_{j \in S_{k}} P_{i j}, \\
= & \sum_{j \in I_{T}} h_{k, j} \cdot P_{i j}+\sum_{j \in S_{k}} P_{i j} .
\end{aligned}
$$

Therefore, for $i \in \mathcal{I}_{T}$, we have shown that $h_{k, i}, i \in \mathcal{I}_{T}$ is a solution to the problem

$$
\sum_{j \in \mathcal{I}_{T} \cup S_{k}} P_{i j} g_{j}=g_{i} \text { subject to } g_{j}=1 \text { for } j \in S_{k} .
$$

Now, we show that $h_{k, i}, i \in \mathcal{I}_{T}$ is the minimal solution to (B1). Suppose that $f$ is another solution; then, $f_{i}=1$ for $i \in S_{k}$, and for $i \in \mathcal{I}_{T}$, we have

$$
f_{i}=\sum_{j \in \mathcal{I}_{T} \cup S_{k}} P_{i j} f_{j}=\sum_{j \in S_{k}} P_{i j}+\sum_{j \in \mathcal{I}_{T}} P_{i j} f_{j} .
$$

Substituting $f_{j}=\sum_{l \in \mathcal{I}_{T} \cup S_{k}} P_{j l} f_{l}$ in the final term,

$$
\begin{aligned}
f_{i}= & \sum_{j \in S_{k}} P_{i j}+\sum_{j \in \mathcal{I}_{T}} P_{i j}\left\{\sum_{l \in S_{k}} P_{j l}+\sum_{l \in \mathcal{I}_{T}} P_{j l} f_{l}\right\} \\
= & \sum_{j \in S_{k}} P_{i j}+\sum_{j \in \mathcal{I}_{T}, l \in S_{k}} P_{i j} P_{j l}+\sum_{j, l \in \mathcal{I}_{T}} P_{i j} P_{j l} f_{l} \\
= & \mathbb{P}\left\{Z_{t+1} \in S_{k} \mid Z_{t}=i\right\}+\mathbb{P}\left\{Z_{t+2} \in S_{k} \mid Z_{t}=i, Z_{t+1} \in \mathcal{I}_{T}\right\} \\
& +\sum_{j, l \in \mathcal{I}_{T}} P_{i j} P_{j l} f_{l} .
\end{aligned}
$$

After repeating the substitution for $f$ in the final term $n$ times, we obtain 


$$
\begin{aligned}
f_{i}= & \mathbb{P}\left\{Z_{t+1} \in S_{k} \mid Z_{t}=i\right\}+\cdots \\
& +\mathbb{P}\left\{Z_{t+n} \in S_{k} \mid Z_{t}=i, Z_{t+1}, \ldots Z_{t+n-1} \in \mathcal{I}_{T}\right\} \\
& +\sum_{j_{1}, \ldots, j_{n} \in \mathcal{I}_{T}} P_{i j_{1}} P_{j_{1} j_{2}} \ldots P_{j_{n-1} j_{n}} f_{j_{n}} .
\end{aligned}
$$

If $f \geq 0$, then the last term on the right is nonnegative, and the remaining terms sum to $\mathbb{P}\left\{Z_{t+r} \in S_{k}\right.$ some $\left.0 \leq r \leq n \mid Z_{t}=i\right\}$ (the probability of hitting $S_{k}$ within $n$ steps). So

$$
\begin{gathered}
f_{i} \geq \lim _{n \rightarrow \infty} \mathbb{P}\left\{Z_{t+r} \in S_{k} \text { some } 0 \leq r \leq n \mid Z_{t}=i\right\} \\
\quad=\mathbb{P}\left\{Z_{t+r} \in S_{k} \text { some } r \geq 0 \mid Z_{t}=i\right\}=h_{k, i} .
\end{gathered}
$$

${ }^{1}$ M. Kubota, "A mechanism for the accumulation of floating marine debris north of Hawaii," J. Phys. Oceanogr. 24, 1059-1064 (1994).

${ }^{2}$ C. Moore, "Synthetic polymers in the marine environment: a rapidly increasing, long-term threat," Environ. Res. 108, 131-139 (2008).

${ }^{3}$ N. Maximenko, J. Hafner, and P. Niiler, "Pathways of marine debris derived from trajectories of Lagrangian drifters," Mar. Pollut. Bull. 65, 51-62 (2012).

${ }^{4}$ E. van Sebille, M. England, and G. Froyland, "Origin, dynamics and evolution of ocean garbage patches from observed surface drifters," Environ. Res. Lett. 7, 044040 (2012).

${ }^{5}$ G. Froyland, K. Padberg, M. England, and A.-M. Treguier, "Detection of coherent oceanic structures via transfer operators," Phys. Rev. Lett. 98, 224503 (2007).

${ }^{6}$ M. Dellnitz, G. Froyland, C. Horenkamp, K. Padberg-Gehle, and A. S. Gupta, "Seasonal variability of the subpolar gyres in the Southern Ocean: A numerical investigation based on transfer operators," Nonlinear Process. Geophys. 16, 655-663 (2009).

${ }^{7}$ V. Rossi, E. Ser Giacomi, C. López, and E. Hernández García, "Hydrodynamic provinces and oceanic connectivity from a transport network help designing marine reserves," Geophys. Res. Lett. 41(8), 2883-2891, doi:10.1002/(ISSN)1944-8007 (2014).

${ }^{8}$ E. Kazantsev, "Unstable periodic orbits and attractor of the barotropic ocean model," Nonlinear processes in Geophysics 5, 193-208 (1998).

${ }^{9}$ E. Kazantsev, "Sensitivity of the attractor of the barotropic ocean model to external influences: Approach by unstable periodic orbits," Nonlinear process. Geophys. 8, 281-300 (2001).

${ }^{10}$ S. Khatiwala, M. Visbeck, and M. Cane, "Accelerated simulation of passive tracers in ocean circulation models," Ocean Modell. 9, 51-69 (2005).

${ }^{11}$ S. Khatiwala, "A computational framework for simulation of biogeochemical tracers in the ocean," Global Biogeochem. Cycles 21, GB3001, doi: 10.1029/2007GB002923 (2007).

${ }^{12} \mathrm{M}$. Dellnitz and O. Junge, "On the approximation of complicated dynamical behavior," SIAM J. Numer. Anal. 36, 491-515 (1999).

${ }^{13}$ M. Demers and L.-S. Young, "Escape rates and conditionally invariant measures," Nonlinearity 19, 377-397 (2006).

${ }^{14}$ Y. Masumoto, H. Sasaki, T. Kagimoto, N. Komori, A. Ishida, Y. Sasai, T. Miyama, T. Motoi, H. Mitsudera, K. Takahashi, et al., "A fifty-year eddyresolving simulation of the world ocean: Preliminary outcomes of OFES (OGCM for the Earth simulator)," J. Earth Simul. 1, 35-56 (2004), see
http://www.jamstec.go.jp/esc/publication/journal/jes_vol.1/pdf/JES1-3.2masumoto.pdf.

${ }^{15}$ H. Sasaki, M. Nonaka, Y. Masumoto, Y. Sasai, H. Uehara, and H. Sakuma, "An eddy-resolving hindcast simulation of the quasiglobal ocean from 1950 to 2003 on the earth simulator," in High Resolution Numerical Modelling of the Atmosphere and Ocean (Springer, 2008) pp. $157-185$.

${ }^{16}$ S. Ulam, A Collection of Mathematical Problems (Interscience, 1979).

${ }^{17}$ C. Hsu, Cell-to-Cell Mapping: A Method of Global Analysis for Nonlinear Systems (Springer-Verlag, New York, 1987).

${ }^{18} \mathrm{G}$. Froyland, "Extracting dynamical behaviour via Markov models," in Nonlinear Dynamics and Statistics: Proceedings of the Newton Institute, Cambridge, 1998, edited by A. Mees (Birkhauser, 2001), pp. 283-324.

${ }^{19}$ C. B. Paris, J. Helgers, E. van Sebille, and A. Srinivasan, "Connectivity modeling system: A probabilistic modeling tool for the multi-scale tracking of biotic and abiotic variability in the ocean," Environ. Modell. Softw. 42, 47-54 (2013).

${ }^{20}$ J. Knauss, Introduction to Physical Oceanography (Waveland Press Inc., 1996).

${ }^{21}$ F. Roquet, C. Wunsch, and G. Madec, "On the patterns of wind-power input to the ocean circulation," J. Phys. Oceanogr. 41, 2328-2342 (2011).

${ }^{22}$ J. Milnor, "On the concept of attractor, the theory of chaotic attractors," Commun. Math. Phys. 99, 177-195 (1985).

${ }^{23}$ J. Norris, Markov Chains (Cambridge University Press, 1998).

${ }^{24} \mathrm{P}$. Koltai, "A stochastic approach for computing the domain of attraction without trajectory simulation," Discrete Contin. Dyn. Syst. 2011, 854-863.

${ }^{25}$ R. Tarjan, "Depth-first search and linear graph algorithms," SIAM J. Comput. 1, 146-160 (1972).

${ }^{26}$ T. Kato, Perturbation Theory for Linear Operators, 2nd ed. (Springer, Berlin, 1995).

${ }^{27}$ P. Deufhard, W. Huisinga, A. Fischer, and C. Schütte, "Identification of almost invariant aggregates in reversible nearly uncoupled Markov chains," Linear Algebra Appl. 315, 39-59 (2000).

${ }^{28} \mathrm{P}$. Deuflhard and M. Weber, "Robust Perron cluster analysis in conformation dynamics," Linear Algebra Appl. 398, 161-184 (2004).

${ }^{29}$ B. Gaveau and L. Schulman, "Multiple phases in stochastic dynamics: Geometry and probabilities," Phys. Rev. E 73, 036124 (2006).

${ }^{30} \mathrm{G}$. Froyland, "On Ulam approximation of the isolated spectrum and eigenfunctions of hyperbolic maps," Discrete Contin. Dyn. Syst. 17, 671-689 (2007).

${ }^{31}$ E. van Sebille, L. M. Beal, and W. E. Johns, "Advective time scales of Agulhas leakage to the North Atlantic in surface drifter observations and the 3D OFES model," J. Phys. Oceanogr. 41, 1026-1034 (2011).

${ }^{32}$ P. Brémaud, Markov Chains: Gibbs Fields, Monte Carlo simulation, and Queues (Springer-Verlag, 1999).

${ }^{33}$ G. Froyland, "Statistically optimal almost-invariant sets," Physica D 200, 205-219 (2005).

${ }^{34}$ J. N. Moum, A. Perlin, J. D. Nash, and M. J. McPhaden, "Seasonal sea surface cooling in the equatorial Pacific cold tongue controlled by ocean mixing," Nature 500, 64-67 (2013).

${ }^{35}$ G. Nelson and L. Hutchings, "The Benguela upwelling area," Progress Oceanogr. 12, 333-356 (1983).

${ }^{36}$ J. Sprintall, S. E. Wijffels, R. Molcard, and I. Jaya, "Direct estimates of the Indonesian Throughflow entering the Indian Ocean: 2004-2006," J. Geophys. Res. 114, C07001, doi:10.1029/2008JC005257 (2009). 